\title{
Perfluoroalkyl and polyfluoroalkyl substance exposure and association with sex hormone concentrations: results from the NHANES 2015- 2016
}

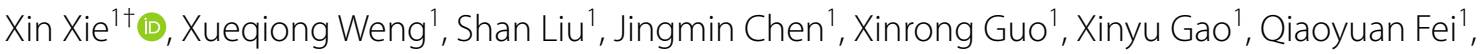 \\ Guang Hao ${ }^{1 *}$, Chunxia Jing ${ }^{1,2^{*}}$ and Liping Feng ${ }^{3 *}$
}

\begin{abstract}
Background: There is increasing global concern regarding the health impacts of perfluoroalkyl and polyfluoroalkyl substances (PFAS), which are emerging environmental endocrine disruptors. Results from previous epidemiological studies on the associations between PFAS exposure and sex hormone levels are inconsistent.
\end{abstract}

Objective: We examined the associations between serum PFAS compounds (PFDeA, PFHXS, PFNA, PFOA, PFOS) and sex hormones, including total testosterone (TT), free testosterone (FT), estradiol (E), and serum hormone binding globulin (SHBG).

Results: After adjusting for potential confounders, PFDeA, PFOS, and PFHxS exposures were significantly associated with increased serum testosterone concentrations in males. PFDeA, PFOA, and PFOS exposures were positively correlated with FT levels in 20-49-year-old women, while PFOS exposure was negatively associated with TT levels in 12-19-year-old girls. PFAS exposure was negatively associated with estradiol levels including: PFDeA in all females, PFHxS, PFNA, PFOS, and PFOA in 12-19-year-old girls, PFNA in women above 50 years, and PFOA in 12-19-year-old boys, while PFDeA and PFOS exposures were positively associated with estradiol levels in these boys. n-PFOS exposure was positively associated with SHBG levels in men older than 20 and in all females.

Conclusions: Using a large cohort of males and females aged from 12 to 80, we found that PFAS exposure appears to disrupt sex hormones in a sex-, age-, and compound-specific manner. Future work is warranted to clarify the causality and mechanisms involved.

Keywords: PFAS, Testosterone, Estradiol, SHGB, NHANES

\footnotetext{
*Correspondence: haoguang2015@hotmail.com; jcxphd@gmail.com; feng0007@mc.duke.edu

${ }^{+}$Xin Xie, Xueqiong Weng and Shan Liu contributed equally to the work 1 Department of Epidemiology, School of Medicine, Jinan University, No.601 Huangpu Ave West, Guangzhou 510632, Guangdong, China ${ }^{3}$ Department of Obstetrics and Gynecology, Duke University School of Medicine, Durham, NC, USA

Full list of author information is available at the end of the article
}

\section{Background}

There is an increasing concern worldwide about exposure to per- and polyfluoralkyl substances (PFAS), a ubiquitous family of chemicals that are highly persistent in the environment and human body [1]. PFAS are synthetic compounds that are suspected endocrine disruptors, compounds capable of causing dysfunction to hormonally regulated body systems [2]. PFAS are characterized by a hydrophobic poly-fluorinated alkyl chain and a polar hydrophilic terminal functional group. PFAS are 
used in multiple commercial applications including surfactants, lubricants, paints, polishes, food packaging, and fire-retarding foams [3, 4]. Certain PFAS compounds are used in the manufacturing of polymers utilized in many consumer products, such as soil-, stain-, grease-, and water-resistant coatings on textiles and carpet; personal care products; and non-stick coatings on cookware, and industrial applications, including the automotive, mechanical, aerospace, chemical, electrical, medical, and building/construction industries [5]. Widespread and continuous daily exposure to PFAS is believed to occur primarily through drinking water, diet, outdoor air, indoor dust, and soil [6].

Human PFAS exposure is associated with many adverse health effects, including endocrine disruption, liver and immune toxicity, and alterations in metabolism and development [7-10]. There is increasing evidence that PFAS exposure is associated with reproductive health issues including a decrease in fertility, reduced fetal growth and birth weight, pregnancy-induced hypertension and preeclampsia, thyroid hormone disruption during pregnancy, and preterm birth [11-15].

PFAS are recognized endocrine disruptors with effects on thyroid hormone disruption [16, 17], metabolism (obesity-promoting) $[18,19]$, and the synthesis of steroid hormone [20]. Reproductive hormones play pivotal roles in both male and female puberty development and are crucial to growth and the functioning of a broad range of tissues, especially reproductive tissues [21]. Testosterone, the primary male sex hormone, is an androgenic steroid with an anabolic effect in humans. Estrogens, the primary female sex hormones, are responsible for the development and regulation of the female reproductive system and secondary female sex characteristics. Sex hormonebinding globulin (SHBG) is the blood transport protein for androgens and estrogens.

Few prior epidemiological studies have investigated the impact of PFAS on the human sex hormones, and the results have been inconsistent. Some studies showed a negative association between PFAS exposure and sex hormones [22-24]. Some studies demonstrated a positive association between PFAS exposure and sex hormones $[25,26]$. PFAS exposure during pregnancy was not associated with age at menarche in a British cohort [27], but PFOA exposure in utero were associated with a later age of menarche in a pregnant Danish cohort [28]. In animals, PFOA exposure has been associated with decreased serum testosterone levels in Leydig cell adenomas [29], increased estradiol levels in rodents [30], and increased steroid hormones in polar bears [31]. In contrast, Behr et al., claimed that PFAS did not affect estrogen or androgen receptor activity or steroidogenesis in human cells [32]. Overall, studies investigating the impact of PFAS on sex hormones are controversial, and most have analyzed only a few PFAS compounds and their effects on children and young adults. Further studies with a larger cohort, broader age groups, and emerging PFAS compounds are warranted.

In this study, we aimed to assess the relationship between exposure to ten PFAS compounds and sex hormones, including total testosterone (TT), free testosterone (FT), estradiol (E), and SHBG using data from the U.S. National Health and Nutrition Examination Survey (NHANES) for 2015-2016, the large-scale populationbased epidemiological study of serum PFAS concentrations, with sufficient power and a broader range of age.

\section{Methods}

\section{Study population}

We used publicly available NHANES data generated through the 2015-2016 survey to analyze the relationship between PFAS exposure and sex hormone concentrations in both males and females. The NHANES is a research program designed to assess the health and nutritional status of adults and children in the United States [33, 34]. The NHANES study protocol is described in detail elsewhere [34-36]. The NHANES agreement has been reviewed and approved by the NCHS Research Ethics Committee [37]. All participants provided written informed consent prior to participation.

Using the unique survey participant identifiers, we linked the database of laboratory test results to other NHANES databases. After the databases were merged, we excluded participants who had missing values from their physical examinations, PFAS and sex steroid hormone measurements, reproductive health or cotinine. After all exclusions, there remained an analytical sample of 1868 participants, including 964 males and 922 females (Fig. 1). The specific introduction of the adjusted covariates and data is in the method of Additional file 1: Materials.

\section{Analysis of serum PFAS concentrations}

Ten perfluoroalkyl and polyfluoroalkyl substances were measured, including perfluorohexane sulfonic acid $(\mathrm{PFHxS})$, perfluorononanoic acid (PFNA), n-perfluorooctanoic acid (n-PFOA), n-perfluorooctane sulfonic acid (n-PFOS), sum of branched perfluorooctanoate isomers (Sb-PFOA), perfluoromethylheptane sulfonic acid isomers (Sm-PFOS), perfluorodecanoic acid (PFDeA), 2-(N-methylperfluoroctanesulfonamido) acetic acid (Me-PFOSA-AcOH), perfluoroundecanoic acid (PFUA), and perfluorododecanoic acid (PFDoA). $\mathrm{n}$-PFOS is a linear PFOS and Sm-PFOS is branched isomers of PFOS. n-PFOA is a linear PFOA [5]. We summed the concentrations of branched and linear isomers of 


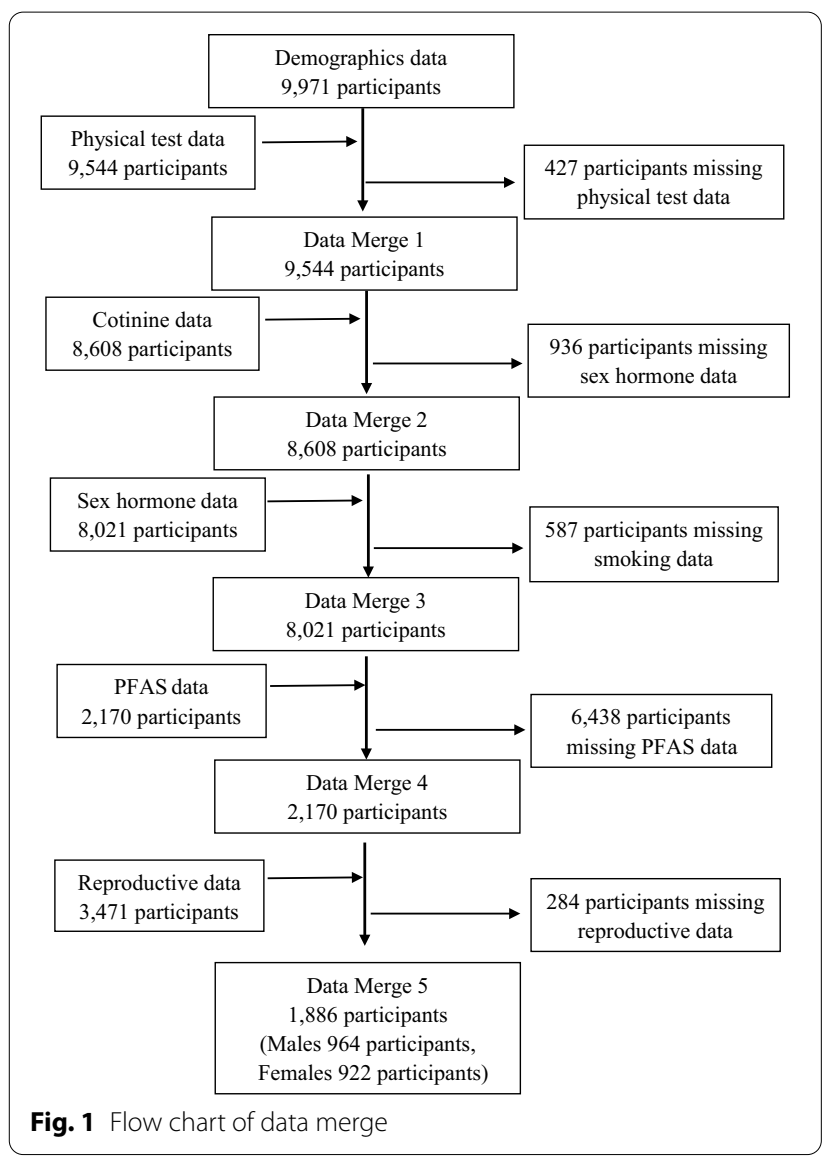

PFOA and PFOS to obtain the "total" concentrations: $\sum \mathrm{PFOA}=\mathrm{n}-\mathrm{PFOA}+\mathrm{Sb}-\mathrm{PFOA}, \sum \mathrm{PFOS}=\mathrm{n}-\mathrm{PFOS}+\mathrm{Sm}-$ PFOS. For statistical analysis, we only included the compounds with detection frequencies above $60 \%$ including PFDeA, PFHxS, PFNA, n-PFOA, n-PFOS, Sm-PFOS.

\section{Outcome assessments}

Testosterone and estradiol are preformed via isotope dilution liquid chromatography tandem mass spectrometry (ID-LC-MS/MS) method, SHBG is based on the reaction of SHBG with immuno-antibodies and chemoluminescence measurements of the reaction products. Refer to the NHANES Laboratory Method Files section for a detailed description of the laboratory methods used [38].

The LODs for TT and E were $0.75 \mathrm{ng} / \mathrm{mL}$ and $2.994 \mathrm{pg} /$ $\mathrm{mL}$, respectively, and the LOD of SHBG was $0.8 \mathrm{nmol} / \mathrm{L}$ [39].

Because direct measurement of FT is impractical in routine practice, we used an online calculator, developed by Professor J.M. Kaufman in the Department of Hormones, Ghent University Hospital, Belgium, to calculate it based on concentrations of TT, SHBG, and albumin [40].

\section{Statistical analysis}

As recommended by NHANES, all analyses included sample weights to account for oversampling nonresponse [41]. Continuous variables were presented as mean and standard deviation (SD), or median and interquartile range if the variables were not normally distributed, while categorical variables were expressed as case $(n)$ and percentage (\%). Outcome variables were checked for normality of distribution, and appropriate transformations were applied when it is necessary. PFAS levels were divided into quartiles (Q1, Q2, Q3, and Q4). The linear regression model was used to analyze the associations between PFAS compounds and sex hormones, and all the models were adjusted for age, race, BMI, education, ratio of family income to poverty, and cotinine. In females, the models were also further adjusted for age at menarche, the use of contraceptives. The regulation, biosynthesis, and functions of sex hormones are different in males and females; therefore, we performed unstratified and sex- and age-stratified analysis. All data were analyzed using STATA (version 14 Stata Corp LP College Station, Texas, USA). Results for which $P<0.05$ were considered significant.

\section{Results}

Basic demographic characteristics of participants of 964 males (51.11\%) and 922 females (48.89\%) are shown in Table 1. We categorized age into three groups: 12-19, $20-49$, and $\geq 50$ years. The overall age distribution was $17.50 \%(n=330)$ between 12 and 19, 41.62\% $(n=785)$ between 20 and 49 , and $40.88 \%(n=771) \geq 50$ years. We classified participants as underweight (BMI below 18.5, 3.45\%), normal weight (BMI between 18.5 and 24.9, $29.06 \%$ ), and overweight (BMI above 24.9, 67.50\%). The distributions of BMIs were similar among males and females. The cohort was racially and ethnically diverse: 19.09\% Mexican American, 12.83\% other Hispanic, $31.55 \%$ Non-Hispanic White, $22.00 \%$ Non-Hispanic Black, and $14.53 \%$ other race. The distribution of education levels was similar among males and females. The median concentration of cotinine is $0.05 \mathrm{ng} / \mathrm{mL}$ in males and $0.03 \mathrm{ng} / \mathrm{mL}$ in females. The ratio of family income to poverty was lower than the standard among $75.82 \%$ of participants. The average age of women at menarche is 12.63 years, and $59.65 \%$ of women have taken contraceptives. For males, the median serum concentrations of TT, E, SHBG, and FT were $392 \mathrm{ng} / \mathrm{dL}, 23.20 \mathrm{pg} / \mathrm{mL}$, $38.32 \mathrm{nmol} / \mathrm{L}$, and $6.91 \mathrm{ng} / \mathrm{dL}$, respectively. For females, the median serum concentrations of TT, E, SHBG, and FT were $20.45 \mathrm{ng} / \mathrm{dL}, 24.75 \mathrm{pg} / \mathrm{mL}, 60.50 \mathrm{nmol} / \mathrm{L}$, and $0.23 \mathrm{ng} / \mathrm{dL}$, respectively. The $\mathrm{E}$ concentrations in females were varied dramatically among age group: $60.30 \mathrm{pg} /$ 
Table 1 Basic demographic characteristics of the participants ( $N=964$ males and 922 females)

\begin{tabular}{|c|c|c|c|}
\hline & Female (\%/P25, P75) & Male (\%/P25, P75) & Total (\%/P25, P75) \\
\hline \multicolumn{4}{|l|}{ Age } \\
\hline $12-19$ & $136(14.75)$ & $194(20.12)$ & $330(17.50)$ \\
\hline $20-49$ & $394(42.73)$ & $391(40.56)$ & $785(41.62)$ \\
\hline$\geq 50$ & $392(42.52)$ & $379(39.32)$ & $771(40.88)$ \\
\hline Menstrual age & $12.63 \pm 1.79$ & - & - \\
\hline \multicolumn{4}{|l|}{ BMI } \\
\hline$<18.5$ & $21(2.28)$ & $44(4.56)$ & $65(3.45)$ \\
\hline $18.5-24.9$ & $260(28.20)$ & $288(29.88)$ & $548(29.06)$ \\
\hline$>24.9$ & $641(69.52)$ & $632(65.56)$ & $1273(67.50)$ \\
\hline \multicolumn{4}{|l|}{ Race } \\
\hline Mexican American & $185(20.07)$ & $175(18.15)$ & 360 (19.09) \\
\hline Other Hispanic & $126(13.67)$ & $116(12.03)$ & $242(12.83)$ \\
\hline Non-Hispanic White & $273(29.61)$ & $322(33.40)$ & $595(31.55)$ \\
\hline Non-Hispanic Black & $221(23.97)$ & $194(20.12)$ & $415(22.00)$ \\
\hline Other Race & $117(12.69)$ & $157(16.29)$ & $274(14.53)$ \\
\hline \multicolumn{4}{|l|}{ Education } \\
\hline Less than 9th grade & $156(17.35)$ & $198(21.27)$ & $354(19.34)$ \\
\hline $9-11$ th grade & $125(13.90)$ & $150(16.11)$ & $275(15.03)$ \\
\hline High school graduate & $168(18.69)$ & $174(18.69)$ & $342(18.69)$ \\
\hline Some college/AA degree & $261(29.03)$ & $229(24.60)$ & $490(26.78)$ \\
\hline College graduate & $189(21.02)$ & $180(19.33)$ & $369(20.16)$ \\
\hline \multicolumn{4}{|c|}{ Ratio of family income to poverty } \\
\hline Under standard & $704(76.36)$ & $726(75.31)$ & $1430(75.82)$ \\
\hline Above standard & $218(23.64)$ & $238(24.69)$ & $456(24.18)$ \\
\hline \multicolumn{4}{|l|}{ Ever taken birth control pills } \\
\hline Yes & $550(59.65)$ & - & - \\
\hline No & $372(40.35)$ & - & - \\
\hline Cotinine $(\mathrm{ng} / \mathrm{mL})$ & $0.03(0.01,0.22)$ & $0.05(0.01,26.20)$ & $0.03(0.01,2.15)$ \\
\hline $\mathrm{TT}(\mathrm{ng} / \mathrm{dL})$ & $20.45(14.10,29.30)$ & $392.00(285.00,517.00)$ & $120.00(20.50,396.00)$ \\
\hline$E(p g / m L)$ & $24.75(6.44,84.50)$ & $23.20(17.80,30.20)$ & $23.60(13.00,37.80)$ \\
\hline SHBG (nmol/L) & $60.50(39.96,90.49)$ & $38.32(26.95,54.48)$ & $46.61(32.36,71.47)$ \\
\hline $\mathrm{FT}(\mathrm{ng} / \mathrm{dL})$ & $0.23(0.15,0.36)$ & $6.91(5.05,9.03)$ & $1.33(0.23,6.95)$ \\
\hline
\end{tabular}

$\mathrm{mL}, 68.10 \mathrm{pg} / \mathrm{mL}$, and $6.29 \mathrm{pg} / \mathrm{mL}$ for $12-19,20-49$, and $\geq 50$ years, respectively (Table 2 ).

$T T$ total testosterone, E estradiol, SHGB serum hormone binding globulin, $F T$ free testosterone

We detected PFDeA, n-PFOA, n-PFOS, Sm-PFOS, PFHxS, and PFNA in the serum of all subjects. By contrast, we only detected Me-PFOSA-AcOH, PFU, and PFDoA in the serum of $39.29 \%, 37.68 \%$, and $2.31 \%$, respectively, of all participants. The serum concentrations of PFAS among participants in this cohort agreed relatively well with those reported among the U.S. general population of adolescents and adults during the same time period [42]. The GM $(95 \% \mathrm{CI})(\mathrm{ng} / \mathrm{mL})$ of the most frequently detected PFAS were: $0.15(0.15-0.16)$ for PFDeA, 1.11 (1.06-1.16) for PFHxS, and 0.60 (0.58-0.62) for PFNA, 1.40 (1.36-1.45) for n-PFOA, 3.30 (3.16-3.44) for n-PFOS, 1.34 (1.29-1.40) for Sm-PFOS. Median concentrations $(\mathrm{ng} / \mathrm{mL})$ and interquartile range (IQR) of PFAS among three age groups in males and females were presented in Table 3 . The serum concentrations of PFAS were higher in older groups $(P<0.001)$, which might be due to the bioaccumulation and longer half-life of PFAS in older populations $[43,44]$.

Among the fully adjusted model for all male participants, we found that TT levels were positively associated with PFDeA at Q3 $(P=0.045)$, PFHxS at Q2 $(P=0.021)$ and Q3 $(P=0.004), n-P F O S$ at Q3 $(P=0.008)$ and Q4 $(P=0.024)$, Sm-PFOS at Q3 $(P=0.013)$ and Q4 $(P<0.001)$, and $\sum$ PFOS at Q3 $(P=0.028)$ and Q4 $(P=0.010)$ levels. The associations between PFAS 
Table 2 Concentrations of sex hormones in different age groups

\begin{tabular}{lccc}
\hline & $\mathbf{1 2 - 1 9}$ years & $\mathbf{2 0 - 4 9}$ years & $\geq \mathbf{5 0}$ years \\
\hline Males & & & \\
Total testosterone & $386.00(231.00,539.00)$ & $418.50(306.00,524.00)$ & $367.00(280.00,497.00)$ \\
Estradiol & $18.90(12.20,25.60)$ & $23.90(18.90,30.20)$ & $24.70(19.00,31.50)$ \\
SHBG & $32.92(22.50,47.05)$ & $32.34(21.71,42.18)$ & $50.80(37.94,70.41)$ \\
Free testosterone & $7.52(4.83,10.23)$ & $8.37(6.59,10.12)$ & $5.44(4.26,6.92)$ \\
Females & & & $16.85(11.50,23.60)$ \\
Total testosterone & $28.30(20.20,36.90)$ & $68.25(15.70,30.10)$ & $6.29(3.73,10.80)$ \\
Estradiol & $60.30(35.00,140.00)$ & $61.59(41.23,97.04)$ & $60.75(40.10,90.11)$ \\
SHBG & $54.39(36.02,75.56)$ & $0.24(0.15,0.38)$ & $0.20(0.13,0.29)$ \\
Free testosterone & $0.36(0.23,0.50)$ & & \\
\hline
\end{tabular}

Total testosterone (ng/dL); estradiol (pg/mL); SHGB (nmol/L); free testosterone $(\mathrm{ng} / \mathrm{dL})$

Table 3 Median and quartile serum concentrations of PFAS in all subjects under covariate status

\begin{tabular}{lllllll}
\hline $\begin{array}{l}\text { Concentration } \\
\text { (ng/mL) }\end{array}$ & PFDeA & PFHxS & PFNA & n-PFOA & n-PFOS & Sm-PFOS \\
\hline Males & & & & & & \\
$12-19$ & $0.10(0.07,0.20)^{* *}$ & $1.00(0.70,1.60)^{* *}$ & $0.50(0.40,0.70)^{* *}$ & $1.30(1.00,1.70)^{* *}$ & $2.40(1.70,3.50)^{* *}$ & $1.00(0.80,1.40)^{* *}$ \\
$20-49$ & $0.01(0.07,0.20)$ & $1.60(1.10,2.60)$ & $0.60(0.40,0.80)$ & $1.80(1.30,2.40)$ & $3.70(2.50,5.80)$ & $1.90(1.30,3.00)$ \\
$\geq 50$ & $0.20(0.07,0.30)$ & $1.80(1.20,2.90)$ & $0.80(0.50,1.10)$ & $2.10(1.40,2.70)$ & $5.60(3.40,9.20)$ & $2.90(1.80,4.40)$ \\
Females & & & & & & \\
$12-19$ & $0.07(0.07,0.20)^{* *}$ & $0.70(0.40,1.30)^{* *}$ & $0.40(0.30,0.60)^{* *}$ & $1.00(0.70,1.40)^{* *}$ & $1.70(1.20,2.30)^{* *}$ & $0.70(0.50,0.90)^{* *}$ \\
$20-49$ & $0.10(0.07,0.20)$ & $0.60(0.40,1.10)$ & $0.40(0.30,0.70)$ & $1.00(0.60,1.60)$ & $2.00(1.20,3.00)$ & $0.80(0.50,1.30)$ \\
$\geq 50$ & $0.20(0.10,0.30)$ & $1.30(0.80,2.20)$ & $0.70(0.50,1.10)$ & $1.80(1.20,2.60)$ & $4.00(2.50,6.50)$ & $1.90(1.00,3.40)$ \\
\hline
\end{tabular}

${ }^{*} P<0.05 ;{ }^{* *} P<0.01$

exposure and FT concentrations resembled the TT results, with $P$ values for PFDeA at Q2, Q3 and Q4 are 0.042, 0.008 and 0.003; PFHxS at Q2, Q3 and Q4 are $0.001,0.003$ and 0.012; n-PFOS at Q2, Q3 and Q4 are 0.025, 0.021 and 0.001; Sm-PFOS at Q3 and Q4 are 0.044 and 0.001; and $\sum$ PFOS at Q3 and Q4 are 0.026 and 0.001 , respectively, for all male participants. There was a linear relationship between TT/FT and PFDeA, n-PFOS, SmPFOS and $\sum$ PFOS $(P<0.05)$ in all subjects (Figs. 2,3$)$. Overall, PFDeA, PFHxS, and PFOS exposures are significantly associated with increased testosterone levels in males.

Furthermore, we observed age specific relationships between PFAS exposure and sex hormone levels. We did not observe the positive association between PFAS exposure and testosterone levels in 12-19-year-old boys. However, among these subjects, PFDeA at Q2 $(P=0.032)$ was positive associated with $\mathrm{E}$ concentrations, while $\mathrm{n}$-PFOA at Q3 $(P=0.022)$ and $\sum$ PFOA at Q3 $(P=0.022)$ were negatively associated with $\mathrm{E}$ concentrations (Additional file 2: Tables S1-S4).

Among 20-49-year-old man, TT levels are positively associated with PFOS including n-PFOS at Q3 levels
$(P=0.046), \mathrm{Sm}-\mathrm{PFOS}$ at Q2 $(P=0.043)$, and $\sum$ PFOS at Q3 $(P=0.027)$. n-PFOS was also positively associated with SHBG levels at Q3 $(P=0.035)$ (Additional file 2: Tables S1-S4).

Sex hormone levels are significantly disrupted by PFAS exposures in older man ( $\geq 50$ years) in this cohort. Similar as the results of all male participants, there was a linear relationship between TT and n-PFOA, $\sum$ PFOA n-PFOS, and $\sum$ PFOS levels and between FT and PFDeA, n-PFOS, and $\sum$ PFOS levels $(P<0.05)$. Specifically, TT levels were positively associated with PFDeA at Q4 levels $(P=0.044)$, PFNA at Q2 $(P=0.048), \mathrm{n}-\mathrm{PFOA}$ at $\mathrm{Q} 3(P=0.040)$ and Q4 $(P=0.015)$, n-PFOS at Q3 $(P=0.035)$ and Q4 $(P=0.039)$, Sm-PFOS at Q3 $(P=0.011), \sum$ PFOA at Q3 $(P=0.041)$ and Q4 $(P=0.016)$, and $\sum$ PFOS at Q3 $(P=0.038)$. FT levels were positively associated with PFDeA at Q3 $(P=0.026)$ and Q4 $(P=0.001)$, PFNA at Q2 $(P=0.035)$ and Q4 $(P=0.043), \mathrm{n}$-PFOA at Q4 $(P=0.019), \mathrm{n}-\mathrm{PFOS}$ at $\mathrm{Q} 2(P=0.024)$ and $\mathrm{Q} 4$ $(P=0.025)$, Sm-PFOS at Q3 $(P=0.009)$, and $\sum$ PFOA at Q4 $(P=0.017)$. E concentrations were positive associated with Sm-PFOS at Q3 $(P=0.021)$ and $\sum$ PFOS at 


\begin{tabular}{|c|c|c|c|c|c|}
\hline$\pi$ & Quartile & Coef $(95 \% \mathrm{Cl})$ & $\begin{array}{c}P \\
\text { value }\end{array}$ & $\begin{array}{l}P \text { for } \\
\text { interaction }\end{array}$ & $\mathrm{R}^{2}$ \\
\hline \multirow[t]{4}{*}{ PFDeA } & Quartile 1 & Ref. & & .024 & .138 \\
\hline & Quartile 2 & $29.92(-11.01,70.85)$ & .152 & & \\
\hline & Quartile 3 & $36.26(0.59,71.93)$ & .045 & & \\
\hline & Quartile 4 & $50.32(-4.05,104.69)$ & .07 & & \\
\hline \multirow[t]{4}{*}{ PFHxS } & Quartile 1 & Ref. & & .211 & .133 \\
\hline & Quartile 2 & $46.67(7.07,86.28)$ & .021 & & \\
\hline & Quartile 3 & $59.47(18.84,100.10)$ & .004 & & \\
\hline & Quartile 4 & $34.21(-9.05,77.67)$ & .121 & & \\
\hline \multirow[t]{4}{*}{ n-PFOS } & Quartile 1 & Ref. & & .003 & .143 \\
\hline & Quartile 2 & $11.17(-24.70,47.04)$ & .541 & & \\
\hline & Quartile 3 & $61.98(16.38,107.57)$ & .008 & & \\
\hline & Quartile 4 & $54.74(7.27,102.20)$ & .024 & & \\
\hline \multirow[t]{4}{*}{ Sm-PFOS } & Quartile 1 & Ref. & & .001 & .145 \\
\hline & Quartile 2 & $37.08(-2.31,76.47)$ & .065 & & \\
\hline & Quartile 3 & $46.61(9.95,83.27)$ & .013 & & \\
\hline & Quartile 4 & $81.02(37.30,124.76)$ & .001 & & \\
\hline \multirow[t]{4}{*}{$\Sigma$ PFOS } & Quartile 1 & Ref. & & .004 & .143 \\
\hline & Quartile 2 & $17.97(-19.25,55.19)$ & .344 & & \\
\hline & Quartile 3 & $49.83(5.52,94.14)$ & .028 & & \\
\hline & Quartile 4 & $62.41(15.26,109.56)$ & .01 & & \\
\hline
\end{tabular}

Fig. 2 Adjusted linear regression model of the associations between PFAS and total testosterone concentrations in all males

Q3 $(P=0.045)$. SHBG levels was positively associated with n-PFOS at Q3 $(P=0.022)$ (Additional file 2: Tables S1-S4).

No other comparisons were statistically significant in this fully adjusted model in males. The null associations were presented in Additional file 2: Tables S1-S4).

In all female participants, the associations between PFAS exposure and testosterone levels are null. Negative associations were found between PFDeA at Q3 and $\mathrm{E}(P=0.035)$ and $\mathrm{n}$-PFOS at $\mathrm{Q} 2$ with SHBG $(P=0.031)$ (Fig. 4).

In 12-19-year-old girls, there were negative associations between TT levels and n-PFOS at Q2 $(P<0.001)$ and $\sum$ PFOS at Q2 $(P=0.001)$ levels. There were also negative associations between E levels and PFHxS at Q2 $(P=0.010)$, PFNA at Q3 $(P=0.041)$, and $\sum$ PFOS at Q2 $(P=0.049)$ levels. The negative relationship between $\mathrm{E}$ and $\mathrm{n}$-PFOA or $\sum$ PFOA was linear $(P<0.05)$ (Additional file 2: Tables S5-S8).

Among 20-49-year-old women, there were positive associations between FT levels and PFDeA at Q2 $(P=0.020)$ and Q3 $(P=0.028)$, n-PFOA $(P=0.044)$, n-PFOS $(P=0.024)$, Sm-PFOS at Q2 $(P=0.023)$ and Q3 $(P=0.031), \sum$ PFOA at Q2 $(P=0.042)$, and $\sum$ PFOS at Q2 $(P=0.003)$ and Q3 $(P=0.021)$ levels (Additional file 2: Tables S5-S8).

In older women ( $\geq 50$ years), E levels was positively associated with PFDeA at Q4 $(P=0.035)$ and this relationship was linear $(P=0.037)$. In contrast, the Q2 and Q4 concentrations of PFNA were negatively correlated with $\mathrm{E}(P=0.003$ and $=0.037$, respectively) (Additional file 2: Tables S5-S8).

No other comparisons were statistically significant in this fully adjusted model in females. The null associations were presented in Additional file 2: Tables S5-S8.

\section{Discussion}

In this study, we examined the association between PFAS exposure and levels of sex hormones in both male and female populations by broad age groups. After adjusting for potential confounders, we demonstrated that higher levels of PFDeA, PFOS, and PFHxS exposure were significantly associated with increased serum testosterone concentrations in males. Interestingly, we found that this association is age-specific and stronger in older man. It is also sex-specific. We observed a null association between PFAS exposure and testosterone levels in all female 


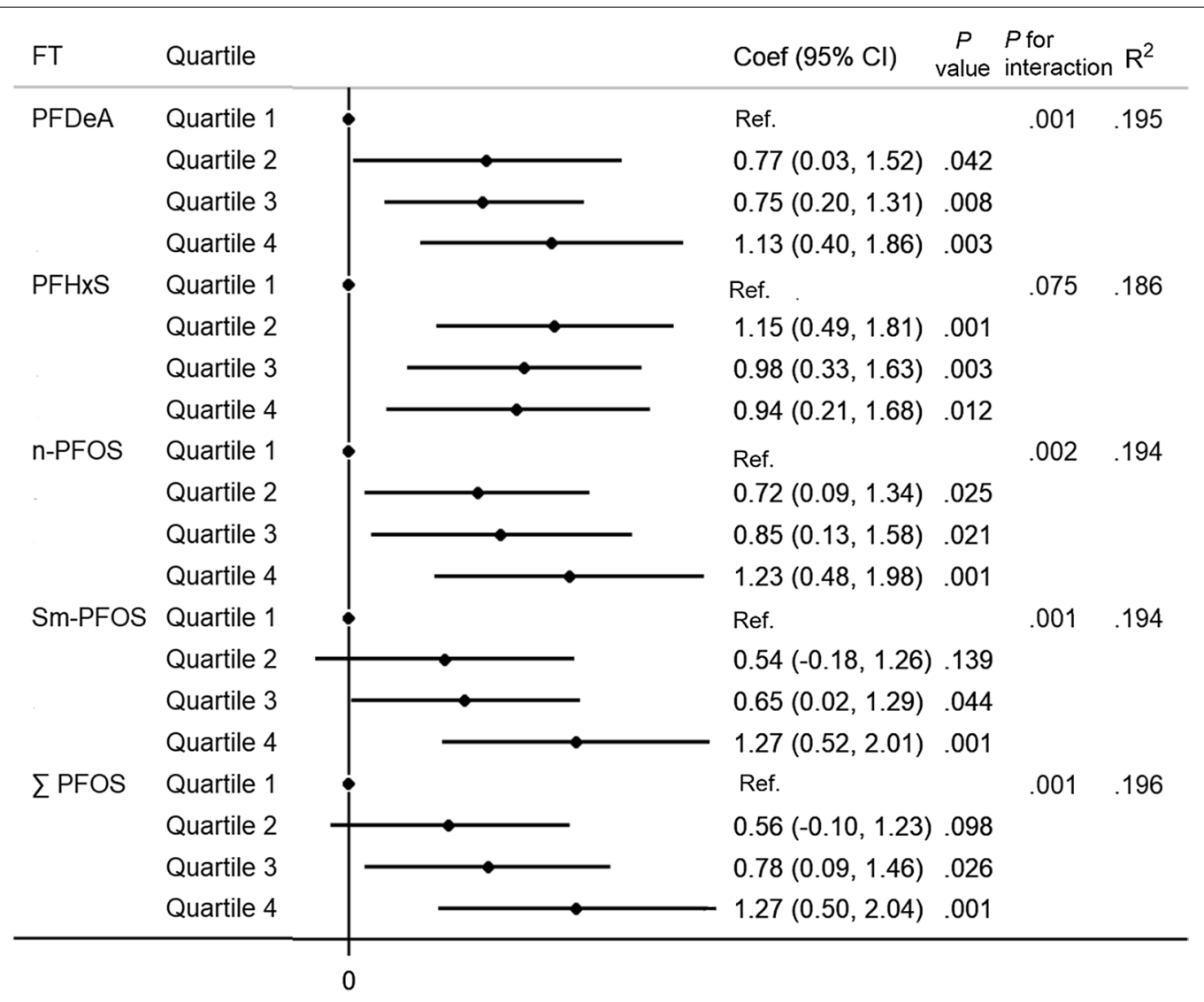

Fig. 3 Adjusted linear regression model of the associations between PFAS and free testosterone concentrations in all males

\begin{tabular}{|c|c|c|c|c|c|}
\hline hormones/PFAS & Quartile & Coef $(95 \% \mathrm{Cl})$ & $\begin{array}{c}P \\
\text { value }\end{array}$ & $\begin{array}{l}P \text { for } \\
\text { interaction }\end{array}$ & $\mathrm{R}^{2}$ \\
\hline \multirow[t]{4}{*}{ E/PFDeA } & Quartile 1 & Ref. & & .086 & .031 \\
\hline & Quartile 2 & $-78.64(-310.37,153.09)$ & .506 & & \\
\hline & Quartile 3 & $-183.04(-353.51,-12.56)$ & .035 & & \\
\hline & Quartile 4 & $-117.92(-285.64,49.70)$ & .168 & & \\
\hline \multirow[t]{4}{*}{ SHBG/n-PFOS } & Quartile 1 & Ref. & & .51 & .079 \\
\hline & Quartile 2 & $-25.52(-48.74,-2.30)$ & .031 & & \\
\hline & Quartile 3 & $-13.78(-37.43,9.87)$ & .253 & & \\
\hline & Quartile 4 & $-9.10(-32.31,14.11)$ & 442 & & \\
\hline
\end{tabular}


subjects and women above 50 years. PFOS exposure was negatively associated with TT levels in 12-19-year-old girls, while PFDeA, PFOA, and PFOS exposures were positively correlate with FT levels in 20-49-year-old women. PFAS exposure was not associated with estradiol levels in all male subjects. However, among 12-19-yearold boys, PFDeA and PFOS exposures were positively associated with estradiol levels, while a negative association was found for PFOA exposure. In females, there were negative associations between PFDeA and estradiol levels in all females, PFHxS, PFNA, PFOS, and PFOA and estradiol levels in 12-19-year-old girls, and PFNA and estradiol levels in women above 50-years. In contrast, PFAS exposure was not associated with estradiol levels in 20-49-year-old women and PFDeA exposure was positively associated with estradiol levels in women above 50 years. The only two significant positive associations between PFAS and SHBG was that for n-PFOS in men older than 20 and in all females. Overall, associations between serum levels of sex hormones and PFAS exposure appeared to be sex-, age-, and compound-specific.

These results are important, because they: (1) provide further evidence of the associations between PFAS exposure and alterations in circulating sex hormone levels and (2) contribute to our understanding of the endocrinedisrupting characteristics of PFAS compounds and which populations may be susceptible to PFAS-induced sex hormone disrupting. Exposure to endocrine disruptors even at low levels, can negatively affect human health such as changes in development and behavior of infants and children and reproductive organs and function; infertility and endometriosis; and disturbances in immune system functions; and increased risk for caner. PFAS exposure has been reported to increase the risk of these endocrine disrupting relevant adverse health effects. Thus, results from our study may bring forth underlying mechanisms of the association between PFAS exposure and adverse reproductive outcomes.

Studies of the relationships between human exposure to PFAS and circulating sex hormones levels in adults and older populations are sparse. Among 12 relevant studies we reviewed and evaluated, 9 studies included populations below 20 years of age and 3 studies investigated this matter among subjects between 20 and 40 years (Additional file 2: Table S9). To the best of our knowledge, ours is the first study to include subjects above 40 years. We observed significant positive correlations between PFDeA, PFHxS, and PFOS exposure and the higher levels of testosterone in males above 50 years. In the same age cohort of males, n-PFOS exposure was positively associated with SHBG levels. However, these associations were null in 12-19-year-old boys, which indicates that PFAS exerts the sex hormone disruption in an age-specific manner. These null associations in young males are in agreement with most previous studies [1, 26, $28,45-48]$ and in disagreement with two previous studies performed in Taiwan which reported an inverse association between PFAS exposure and testosterone levels [24, 25]. Lopez-Espinosa et al. reported a negative association between PFOA exposure and total testosterone in boys aged 6-9 years [49]. The exposure levels of PFOA in this large cohort of boys were high, with a median concentration of $34.8 \mathrm{ng} / \mathrm{mL}$. Conversely, Maisonet et al. reported a positive association between PFOA exposure $(>4.1 \mathrm{ng} / \mathrm{mL})$ and total testosterone in 72 girls with an average age of 15 [45], while we observed a positive association between PFOA exposure and free testosterone levels in 20-49-year-old women. Testosterone disruptions upon PFAS exposures were not observed in women above 50 years, which indicates that the endocrine disruption of PFAS is also sex-specific. The endocrine disruption can also be age-specific. As individuals age, there is a decline in the peripheral levels of estrogen and testosterone and an increase in sex hormone-binding globulin [50]. Menopausal age and the age of puberty can cause changes in female hormone levels. In addition, possible hormone therapy in the post-menopause may affect its own hormone levels. These effects depend of type, dose and administration type, and with age and atherosclerosis stages [51].

Moreover, we are the first group to demonstrate a link between PFDeA and PFHxS exposures and testosterone levels. No such correlations were found in previous studies for PFHxS [22, 25, 45, 46, 48, 49] and PFDeA [25, 46, 48]. Our results showed that PFDeA and PFHxS exposures are positively associated with serum testosterone concentrations in men above 50 years and a positive relationship between PFDeA exposure and free testosterone levels in 20-49-year-old women. The differences in results between our study and the previous studies primarily are contributed by the age differences in these study cohorts given that the positive correlations were only found in older subjects in our study and the null correlations are consistent with previous studies in younger populations. Although the PFAS concentrations in this cohort are at the lower end of the range, as reported by the previous 12 studies, the exposure levels are representative of those in the U.S. population (Additional file 3).

Due to limited evidence, it is difficult to present plausible explanations for these associations. In general, environmental endocrine-disrupting chemicals have antiandrogenic effects that are mediated by mechanisms such as interference with the androgen receptor, androgen production, or metabolism or signaling in the hypothalamic-pituitary-gonadal axis. PFOA or PFOS may interfere with androgen production through down-regulation 
of cytochrome P450 superfamily 11 (CYP11A1) production and inhibition of $3 \beta-H S D 1[20,52]$ and CYP17A1 [52] enzyme activities. CYP11A1 catalyzes conversion of cholesterol to pregnenolone, and this is the first reaction in the process of steroidogenesis in all mammalian tissues [53]. In addition, PFOA or PFOS are cytotoxic to Leydig cells, which are responsible for androgen biosynthesis $[20,52,54]$. These in vitro and in vivo studies suggest a negative correlation between PFOS and PFOA with total testosterone levels in males; however, these results may be due to the high doses used as we observed positive correlations between PFAS exposure and total testosterone levels in this cohort of males. Furthermore, it has been established that PFAS have an affinity for proteins and can bind to albumin, and it is estimated that $90 \%$ of PFOA in serum is bound to albumin [55]. PFOS can bind to SHBG, although with low affinity, and can displace testosterone at very high concentrations [56]. Therefore, to fully understand the endocrine-disrupting properties of PFAS, we must examine the effects of PFAS on SHBG and free testosterone levels. In agreement with several other labs, we demonstrated no associations between PFAS exposure and serum SHBG levels [24, 26, 28, 45-47, 57]. Joensen et al. reported that SHBG levels were positively correlated with PFOS exposure in 247 young males [22], while Tsai reported a negative correlation with PFOA in 330 young females [24]. We observed a positive correlation between n-PFOS levels and SHBG concentrations in men older than 20 and in all females, no other PFAS exposure was associated with SHBG levels. This observation provides partial explanation of the resembled association between PFAS compounds and total testosterone and free testosterone levels in this cohort. There are few studies that have examined the association between PFAS exposure and free testosterone levels and reported no association between PFAS compounds and free testosterone $[22,24,26]$ with the exception of a negative association between PFOS and free testosterone in 247 males with an average age of 19 years [22]. Subjects in all three studies were less than 30 years.

The reverse relationship between PFAS exposure and serum estradiol levels observed in females in this study is consistent with a previous study in a female cohort $(n=178)$ aged 25-35 years [58]. PFOS and PFHxS exposure were also negatively associated with estradiol in a case-control study in 240 females aged $20-40$ years [48]. These associations were not found in males and/or younger populations for PFOA [22, 24-26, 46-49, 57], or PFOS [26, 46, 47], while we found a positive correlation for PFOS and a negative association for PFOA with estradiol levels in boys 12-19 years. For 12-19-year-old girls, PFOA and PFOS exposures were associated with lower levels of estradiol. Results from several previous studies indicate an inverse relationship between PFOS and estradiol in males [22] and younger aged subjects [49, 57]. One study showed positive associations between exposure to PFOS and PFNA and estradiol levels in girls aged 10-15 years [25]. PFNA and PFHxS levels were not associated with serum estradiol concentrations in other studies $[22,25,46,48,49,58]$, but an inverse association was demonstrated in our study. For the first time, we found that PFDeA exposure was positively associated with estradiol levels in boys 12-19 years and a negative association in all females. The inconsistency between studies could be caused by multiple factors, such as exposure levels, sample size, sex, confounder factors, and age; however, based on our analysis, sex and age seem to be the key determining factors. Overall, PFAS exposure appears to be associated with a decrease in serum estradiol concentration in female of reproductive age. The age- and sex-specific associations between PFAS and sex hormones are striking and warrants further investigations.

Plausible explanations for our finding of a correlation between increased PFAS exposure and decreased estradiol levels include: 1) a decreased follicle stimulating hormone (FSH) in females associated with PFOA exposure [24], which would lead to a negative feedback for estrogen biosynthesis in the ovaries; 2) inhibition of steroidogenic enzyme activity such as aromatase [59] and hydroxysteroid-17-beta dehydrogenase 1 (17 $\beta$-HSD3) [20], by PFAS. Aromatase, also called estrogen synthetase or estrogen synthase, is an enzyme responsible for a key step in the biosynthesis of estrogens, and 17 $\beta$-HSD3 catalyzes the last step in estrogen activation; 3 ) the ability of PFAS to act as an estrogen receptor agonist [32, 59], which can result in a negative feedback for estrogen biosynthesis.

The strengths of our study include the large sample size, broad age group and nationally representative nature of NHANES. NHANES, which examines a nationally representative sample of 5000 persons each year. These persons are located in counties across the country, 16 of which are visited each year. Our study cohort is racially and ethnically diverse. The NHANES interview and examination obtained comprehensive demographic and health measures including reproductive health such as age at menarche and use of contraceptives which can influence sex hormones levels in females. Thus, we were able to adjust for several important covariates that are associated with sex hormones and PFAS levels. The methods for measuring serum sex hormones and PFAS meet the national standards, and the implementation of rigorous and well-defined quality control procedures helped to ensure data accuracy. Moreover, our study measured both linear and branched PFOA and PFOS. Electrochemical fluorinated (ECF) and telomerization 
are the two major methods used to produce PFAS. ECF method has historically been used to produce the two dominant compounds, PFOA and PFOS. The typical composition of PFOA and PFOS produced by ECF were $\sim 70 \%$ linear ( $\mathrm{n}$-PFOA and $\mathrm{n}$-PFOS) and $30 \%$ branched isomers (br-PFOA and br-PFOS). Br-PFOA and br-PFOS are generally excreted faster than the linear isomers. The exception is Sm-PFOS, the PFOS isomer containing a perfluorinated methyl group, whose half-life is three times longer than n-PFOS.

There are several limitations to this study. First, the analysis is based on a single measurement of PFAS exposure, which is not a perfect measure of given that human health effects are most likely associated with long-term, low-dose exposure. However, all the PFAS compounds examined in this study are persistent in the human body. For example, the biological half-lives for PFOA, PFOS, PFHxS, PFNA, and PFDeA are 2.1 to $10.1,3.3$ to $27,4.7$ to 35 years [43, 60-62], 1.5 to 4.3 , and 4.3 to 12 years, respectively. Second, we were not able to obtain information about menopause status and hormone therapy in the post-menopause women. Reproductive hormone levels in females varies dramatically by menopause status that was not included in NHANES database. However, the serum estradiol concentrations in females $\leq 50$ and $\geq 50$ years likely consistent with those of premenopausal and postmenopausal females, respectively, which mitigates the lack of status in our cohort. Third, a major limitation of NHANES is that it is not geographically representative of the United States, because the two teams could only visit a total of 16 locations per year; it is impossible to achieve a good geographic spread. Although this cohort is racially and ethnically diverse, it is relatively over weight and poor compared to the general population. Fourth, potential confounders/covariates, such as sample collection time, is not available for this study population, Finally, the causalities cannot be determined due to the crosssectional nature of the associations reported here. Future work needs to replicate the associations found and elucidate the mechanisms involved.

\section{Conclusion}

We found that PFAS levels are significantly associated with increased serum testosterone levels in older men and seem to be associated with decreased serum estradiol concentrations in females in this NHANES cohort. Overall, the consistencies and discrepancies between our findings and all other relevant studies are sex-, age-, and compounds-specific. The range of exposure levels is relatively large among all studies, and the length of half-lives of PFAS compounds is much shorter in younger populations. Additional studies should focus on the mechanisms by which PFAS disrupt sex hormones in both males and females.

\begin{abstract}
Abbreviations
E: Estradiol; FT: Free Testosterone; NHANES: National Health and Nutrition Examination Survey; n-PFOA: N-Perfluorooctanoic acid; n-PFOS: N-Perfluorooctane sulfonic acid; PFAS: Perfluoroalkyl and polyfluoroalkyl substances; PFDeA: Perfluorodecanoic acid; PFHxS: Perfluorohexane sulfonic acid; PFNA: Perfluorononanoic acid; SHBG: Serum hormone binding globulin; Sm-PFOS: Perfluoromethylheptane sulfonic acid isomers; TT:Total testosterone.
\end{abstract}

\section{Supplementary Information}

The online version contains supplementary material available at https://doi. org/10.1186/s12302-021-00508-9.

Additional file 1. Additional methods.

Additional file 2. Additional tables.

Additional file 3. Additional methods.

\section{Acknowledgements}

This study analyzed using the data provided by the National Health and Nutrition Examination Survey (2015-2016) which is used in epidemiological studies and health sciences research to help develop sound public health policy, direct and design health programs and services, and expand the health knowledge for the nation.

\section{Authors' contributions}

XX and GH: Conceptualization, Methodology, Software, Writing-original draft preparation, writing-reviewing and editing. XW and SL: software, data curation, resources, substantively revise. JC and XG: investigation, resources. XG and QF: software, validation. LF and CJ: validation, supervision, writing-reviewing and editing, project administration. All authors read and approved the final manuscript.

\section{Funding}

This research did not receive any specific grant from funding agencies in the public, commercial, or not-for-profit sectors.

Availability of data and materials

The data used in the article can be downloaded for free in NHANES.

\section{Declarations}

\section{Ethics approval and consent to participate}

The NHANES agreement has been reviewed and approved by the NCHS Research Ethics Committee. All participants provided written informed consent prior to participation.

\section{Consent for publication}

All participants provided written informed consent prior to participation.

\section{Competing interests}

The authors report no conflicts of interest.

\section{Author details}

'Department of Epidemiology, School of Medicine, Jinan University, No.601 Huangpu Ave West, Guangzhou 510632, Guangdong, China. ${ }^{2}$ Guangdong Key Laboratory of Environmental Exposure and Health, Jinan University, Guangzhou 510632, Guangdong, China. ${ }^{3}$ Department of Obstetrics and Gynecology, Duke University School of Medicine, Durham, NC, USA. 
Received: 17 March 2021 Accepted: 28 May 2021

Published online: 06 June 2021

\section{References}

1. Bach CC et al (2016) Perfluoroalkyl and polyfluoroalkyl substances and measures of human fertility: a systematic review. Crit Rev Toxicol 46(9):735-755

2. Talsness CE et al (2009) Components of plastic: experimental studies in animals and relevance for human health. Philos Trans R Soc Lond B Biol Sci 364(1526):2079-2096

3. D'Agostino LA, Mabury SA (2014) Identification of novel fluorinated surfactants in aqueous film forming foams and commercial surfactant concentrates. Environ Sci Technol 48(1):121-129

4. Rotander A et al (2015) Novel fluorinated surfactants tentatively identified in firefighters using liquid chromatography quadrupole time-offlight tandem mass spectrometry and a case-control approach. Environ Sci Technol 49(4):2434-2442

5. NHANES. Perfluoroalkyl and Polyfluoroalkyl. 2015-2016; https://wwwn.cdc. gov/Nchs/Nhanes/2015-2016/PFAS I.htm.

6. Fromme $\mathrm{H}$ et al (2009) Perfluorinated compounds-exposure assessment for the general population in Western countries. Int J Hyg Environ Health 212(3):239-270

7. Rappazzo KM (2017) Exposure to perfluorinated alkyl substances and health outcomes in children: a systematic review of the epidemiologic literature. Int J Environ Res Public Health 14:7

8. Liew Z, Goudarzi H, Oulhote Y (2018) Developmental Exposures to Perfluoroalkyl Substances (PFASs): an update of associated health outcomes, Curr Environ Health Rep 5(1):1-19

9. Stein CR, Savitz DA, Dougan M (2009) Serum levels of perfluorooctanoic acid and perfluorooctane sulfonate and pregnancy outcome. Am J Epidemiol 170(7):837-846

10. Huang MC et al (2019) Toxicokinetics of perfluorobutane sulfonate (PFBS), perfluorohexane-1-sulphonic acid (PFHxS), and perfluorooctane sulfonic acid (PFOS) in male and female Hsd: Sprague Dawley SD rats after intravenous and gavage administration. Toxicol Rep 6:645-655

11. Wikström S et al (2020) Maternal serum levels of perfluoroalkyl substances in early pregnancy and offspring birth weight. Pediatric Res 87(6):1093-1099

12. Wikstrom S et al (2019) Early pregnancy serum levels of perfluoroalkyl substances and risk of preeclampsia in Swedish women. Sci Rep 9(1):9179

13. Sagiv SK et al (2018) Early-pregnancy plasma concentrations of perfluoroalkyl substances and birth outcomes in project viva: confounded by pregnancy hemodynamics? Am J Epidemiol 187(4):793-802

14. Huang R et al (2019) Prenatal exposure to perfluoroalkyl and polyfluoroalkyl substances and the risk of hypertensive disorders of pregnancy. Environ Health 18(1):5

15. Wang Y et al (2016) Prenatal Exposure to Perfluorocarboxylic Acids (PFCAs) and Fetal and postnatal growth in the Taiwan maternal and infant cohort study. Environ Health Perspect 124(11):1794-1800

16. Blake BE et al (2018) Associations between longitudinal serum perfluoroalkyl substance (PFAS) levels and measures of thyroid hormone, kidney function, and body mass index in the Fernald Community Cohort. Environ Pollut 242(Pt A):894-904

17. Caron-Beaudoin E et al (2019) Exposure to perfluoroalkyl substances (PFAS) and associations with thyroid parameters in First Nation children and youth from Quebec. Environ Int 128:13-23

18. Lauritzen $\mathrm{HB}$ et al (2018) Prenatal exposure to persistent organic pollutants and child overweight/obesity at 5-year follow-up: a prospective cohort study. Environ Health 17(1):9

19. Jain RB, Ducatman A (2019) Roles of gender and obesity in defining correlations between perfluoroalkyl substances and lipid/lipoproteins. Sci Total Environ 653:74-81

20. Zhao B et al (2010) Inhibition of human and rat 3beta-hydroxysteroid dehydrogenase and 17beta-hydroxysteroid dehydrogenase 3 activities by perfluoroalkylated substances. Chem Biol Interact 188(1):38-43

21. Kjeldsen LS, Bonefeld-Jorgensen EC (2013) Perfluorinated compounds affect the function of sex hormone receptors. Environ Sci Pollut Res Int 20(11):8031-8044
22. Joensen UN et al (2013) PFOS (perfluorooctanesulfonate) in serum is negatively associated with testosterone levels, but not with semen quality, in healthy men. Hum Reprod 28(3):599-608

23. Lopez-Espinosa MJ et al (2011) Prenatal exposure to organochlorine compounds and birth size. Pediatrics 128(1):e127-e134

24. Tsai MS et al (2015) Association between perfluoroalkyl substances and reproductive hormones in adolescents and young adults. Int J Hyg Environ Health 218(5):437-443

25. Zhou Y et al (2016) Association of perfluoroalkyl substances exposure with reproductive hormone levels in adolescents: by sex status. Environ Int 94:189-195

26. Petersen MS et al (2018) Reproductive Function in a Population of Young Faroese Men with Elevated Exposure to Polychlorinated Biphenyls (PCBs) and Perfluorinated Alkylate Substances (PFAS). Int J Environ Res Public Health 15:9

27. Christensen KY et al (2011) Exposure to polyfluoroalkyl chemicals during pregnancy is not associated with offspring age at menarche in a contemporary British cohort. Environ Int 37(1):129-135

28. Kristensen SL et al (2013) Long-term effects of prenatal exposure to perfluoroalkyl substances on female reproduction. Hum Reprod 28(12):3337-3348

29. Cook JC et al (1992) Induction of Leydig cell adenomas by ammonium perfluorooctanoate: a possible endocrine-related mechanism. Toxicol Appl Pharmacol 113(2):209-217

30. Biegel LB et al (1995) Effects of ammonium perfluorooctanoate on Leydig cell function: in vitro, in vivo, and ex vivo studies. Toxicol Appl Pharmacol 134(1):18-25

31. Lau C et al (2007) Perfluoroalkyl acids: a review of monitoring and toxicological findings. Toxicol Sci 99(2):366-394

32. Behr AC et al (2018) Perfluoroalkylated substances (PFAS) affect neither estrogen and androgen receptor activity nor steroidogenesis in human cells in vitro. Toxicol Lett 291:51-60

33. NHANES. Introduction to NHANES. 2015-2016. https://www.cdc.gov/ nchs/data/nhanes/nhanes_13_14/NHANES_Overview_Brochure.pdf.

34. NHANES. The National Health and Nutritional Examination Survey (NHANES). 2015-2016; http://russlopez.com/blog/2012/04/30/the-natio nal-health-and-nutritional-examination-survey-nhanes/.

35. NHANES. NHANES Tutorials. 2015-2016; https://wwwn.cdc.gov/nchs/ nhanes/tutorials/default.aspx.

36. NHANES. About the National Health and Nutrition Examination Survey. 2015-2016; https://www.cdc.gov/nchs/nhanes/about_nhanes.htm.

37. NHANES. NCHS Research Ethics Review Board Approval*. 2015-2016: https://www.cdc.gov/nchs/nhanes/irba98.htm.

38. NHANES. NHANES LPM. 2015-2016; https://wwwn.cdc.gov/nchs/data/ nhanes/2015-2016/manuals/2016_MEC_Laboratory_Procedures_ Manual.pdf.

39. NHANES. Sex Steroid Hormone - Serum. 2015-2016; https://wwwn.cdc gov/Nchs/Nhanes/2015-2016/TST_I.htm.

40. Fiers, T. and J.M. Kaufman. Free \& Bioavailable Testosterone calculator 2020; http://www.issam.ch/freetesto.htm.

41. NHANES. Demographic Variables. 2015-2016; https://wwwn.cdc.gov/ Nchs/Nhanes/2015-2016/DEMO_I.htm.

42. Prevention, C.f.D.C.a. Fourth National Report on Human Exposure to Environmental Chemicals Updated Tables. 2017; https://www.cdc.gov/exposurere port/pdf/FourthReport_UpdatedTables_Volume1_Jan2017.pdf.

43. Zhang $Y$ et al (2013) Biomonitoring of perfluoroalkyl acids in human urine and estimates of biological half-life. Environ Sci Technol 47(18):10619-10627

44. Fu J et al (2016) Occurrence, temporal trends, and half-lives of perfluoroalkyl acids (PFAAs) in occupational workers in China. Sci Rep 6:38039

45. Maisonet $\mathrm{M}$ et al (2015) Prenatal exposure to perfluoroalkyl acids and serum testosterone concentrations at 15 years of age in female ALSPAC study participants. Environ Health Perspect 123(12):1325-1330

46. Joensen UN et al (2009) Do perfluoroalkyl compounds impair human semen quality? Environ Health Perspect 117(6):923-927

47. Vested A et al (2013) Associations of in utero exposure to perfluorinated alkyl acids with human semen quality and reproductive hormones in adult men. Environ Health Perspect 121(4):453-458

48. Zhang S et al (2018) Association of perfluoroalkyl and polyfluoroalkyl substances with premature ovarian insufficiency in chinese women. J Clin Endocrinol Metab 103(7):2543-2551 
49. Lopez-Espinosa MJ et al (2016) Perfluoroalkyl substances, sex hormones, and insulin-like growth factor-1 at 6-9 years of age: a crosssectional analysis within the c8 health project. Environ Health Perspect 124(8):1269-1275

50. Chahal HS, Drake WM (2007) The endocrine system and ageing. J Pathol 211(2):173-180

51. Bejan-Angoulvant T, Arnal JF (2019) Endogenous and exogenous estrogens. Presse Med 48(11 Pt 1):1244-1248

52. Lu H et al (2019) Effects of perfluorooctanoic acid on stem Leydig cell functions in the rat. Environ Pollut 250:206-215

53. Kraugerud $\mathrm{M}$ et al (2011) Perfluorinated compounds differentially affect steroidogenesis and viability in the human adrenocortical carcinoma (H295R) in vitro cell assay. Toxicol Lett 205(1):62-68

54. Eggert A et al (2019) The effects of perfluorooctanoic acid (PFOA) on fetal and adult rat testis. Reprod Toxicol 90:68-76

55. Han X et al (2003) Binding of perfluorooctanoic acid to rat and human plasma proteins. Chem Res Toxicol 16(6):775-781

56. Jones PD et al (2003) Binding of perfluorinated fatty acids to serum proteins. Environ Toxicol Chem 22(11):2639-2649

57. Itoh S et al (2016) Association of perfluoroalkyl substances exposure in utero with reproductive hormone levels in cord blood in the Hokkaido Study on Environment and Children's Health. Environ Int 94:51-59
58. Barrett ES et al (2015) Perfluoroalkyl substances and ovarian hormone concentrations in naturally cycling women. Fertil Steril 103(5):1261-1270

59. Du YB et al (2013) Endocrine and inflammatory factors and endometriosis-associated infertility in assisted reproduction techniques. Arch Gynecol Obstet 287(1):123-130

60. Li Y et al (2018) Half-lives of PFOS, PFHXS and PFOA after end of exposure to contaminated drinking water. Occup Environ Med 75(1):46-51

61. Olsen GW et al (2007) Half-life of serum elimination of perfluorooctanesulfonate, perfluorohexanesulfonate, and perfluorooctanoate in retired fluorochemical production workers. Environ Health Perspect 115(9):1298-1305

62. Worley RR et al (2017) Per- and polyfluoroalkyl substances in human serum and urine samples from a residentially exposed community. Environ Int 106:135-143

\section{Publisher's Note}

Springer Nature remains neutral with regard to jurisdictional claims in published maps and institutional affiliations.

\section{Submit your manuscript to a SpringerOpen ${ }^{\odot}$ journal and benefit from:}

- Convenient online submission

- Rigorous peer review

- Open access: articles freely available online

- High visibility within the field

- Retaining the copyright to your article

Submit your next manuscript at $\boldsymbol{\nabla}$ springeropen.com 